\title{
Charateristics, clinical features, and animal contact history of COVID-19 patients in Leishenshan Hospital, Wuhan China
}

\author{
Yufeng YUAN ${ }^{1}$, Zhen YANG ${ }^{2}$, Bilong FENG ${ }^{1}$, Chang Cai ${ }^{3}$, Junxia SONG ${ }^{4}$, and Likai LIN ${ }^{1}$ \\ ${ }^{1}$ Wuhan University Zhongnan Hospital \\ ${ }^{2}$ Nanjing Agricultural University \\ ${ }^{3}$ Zhejiang $\mathrm{A}$ and $\mathrm{F}$ University \\ ${ }^{4}$ Food and Agriculture Organization of the United Nations
}

June 5, 2020

\begin{abstract}
Leishenshan Hospital was an urgently built filed hospital in Wuhan only for treating coronavirus disease 2019 (COVID-19) patients. From the 8th of February it received the first cohort of patients until the closure day, 15th of April, it totally accepted 2011 COVID-19 patients including 20.9\% severe and $79.1 \%$ non-severe cases with a case fatality rate (CFR) $2.2 \%$ (95\%CI 1.6-3.0\%). Totally 1917 (95.3\%, 95\%CI 94.3-96.2) patients were cured with a recovery rate of $86.2 \%$ in severe group and 100.0 $\%$ in non-severe group. On the 8th of April 2020, Wuhan city eased the lockdown policy after 76 days of the most restrict measurement ever. Herein, we collected 509 medical records from Leishenshan hospital to share some commonly interested COVID-19 clinical data.
\end{abstract}

\section{Short Communication}

Leishenshan Hospital was an urgently built field hospital in Wuhan only for treating coronavirus disease 2019 (COVID-19) patients. From February to April 2020, it totally accepted 2011 COVID-19 patients including $20.9 \%$ severe and $79.1 \%$ non-severe cases. At present, it is closed with a case fatality rate (CFR) is $2.2 \%(95 \%$ CI $1.6-3.0 \%)$. Totally $1917(95.3 \%, 95 \%$ CI $94.3-96.2 \%)$ patients were cured with a recovery rate of $86.2 \%$ in severe group and $100 \%$ in non-severe group. On the $8^{\text {th }}$ of April 2020, Wuhan city eased the lockdown policy after 76 days of the most restrict measurement ever. Herein, we collected 509 medical records from Leishenshan hospital to share some commonly interested COVID-19 clinical data. All extracted and summarised data are showed in table 1.

The origin of the patients covered all 13 districts in Wuhan city. In our records, $53.2 \%$ (95\%CI 48.8-57.6\%) of the COVID-19 patients were female, which is slightly higher than Nanshan Zhong et al . (Guan et al., 2020) (41.9\%, 95\%CI 38.9-44.9\%) and the Chinese Center for Disease Control and Prevention(Novel Coronavirus Pneumonia Emergency Response Epidemiology, 2020) reported (48.6\%, 95\%CI 48.1-49.0\%) reported. Most of the patients distributed in the age groups $>50$ (66.4\%, 95 CI 62.1-70.5\%) which was consistent with large scale surveillance reports from China (Guan et al., 2020; Novel Coronavirus Pneumonia Emergency Response Epidemiology, 2020). Sensory impairment as a clinical symptom got our attention, since 19.5\% of them experienced total or partial taste loss and 7.5\% experienced both smell and taste impairment. There were 42 pet owners in our cases, which only accounted for a small proportion (8.5\%, 95\%CI 6.0-11.0\%). Most of them (25) are raising dogs, ten are raising cats, and one of each in petting chinchillidae, hamster, tortoise, parrot, pigeon, crested myna and goldfish. 23 patients had animal contact history within the 14 days prior to showing clinical symptoms including 12 people contacted with fishes, 10 with chickens and one with bullfrogs. Ten of the patients were doing animal related occupation such as veterinarians, live animal 
market workers, slaughterhouse workers, livestock farmers and farm workers. Animal contact history did not play an important role in COVID-19 positivity in our study, even though more evidences showed that cat and dogs of COVID-19 patients could be infected.(Shi et al., 2020; Sit et al., 2020) It is recommended that follow-up checking on pets in the families of COVID-19 patients was essential.

Exposure history records identified 257 patients with known sources of infection, of whom $69.3 \%$ (95\%CI 63.2-74.8\%) presented fever and actively sought for a COVID-19 test. A total of 46 patients $(17.9 \%, 95 \%$ CI 13.41-23.14\%) experienced no unwellness but were identified through COVID-19 monitoring implementation. They were either asymptomatic carriers or in their incubation period, if were not identified, would fuel the pandemic.

\section{Declaration of interests}

All authors declare no competing interests.

\section{Ethical Statement}

Ethical Statement is not applicable since the questionnaires from patients has been gathered.

\section{Data availability statement}

Data available on request from the authors.

\section{References}

Guan, W. J., Ni, Z. Y., Hu, Y., Liang, W. H., Ou, C. Q., He, J. X., . . . China Medical Treatment Expert Group for, C. (2020). Clinical Characteristics of Coronavirus Disease 2019 in China. N Engl J Med . doi:10.1056/NEJMoa2002032

Novel Coronavirus Pneumonia Emergency Response Epidemiology, T. (2020). [The epidemiological characteristics of an outbreak of 2019 novel coronavirus diseases (COVID-19) in China]. Zhonghua Liu Xing Bing Xue Za Zhi, 41 (2), 145-151. doi:10.3760/cma.j.issn.0254-6450.2020.02.003

Shi, J., Wen, Z., Zhong, G., Yang, H., Wang, C., Huang, B., . . . Bu, Z. (2020). Susceptibility of ferrets, cats, dogs, and other domesticated animals to SARS-coronavirus 2. Science . doi:10.1126/science.abb7015

Sit, T. H. C., Brackman, C. J., Ip, S. M., Tam, K. W. S., Law, P. Y. T., To, E. M. W., . . . Peiris, M. (2020). Infection of dogs with SARS-CoV-2. Nature . doi:10.1038/s41586-020-2334-5

\section{Hosted file}

2020-06-05 Comment-Table1.docx available at https://authorea.com/users/330340/articles/ 457151-charateristics-clinical-features-and-animal-contact-history-of-covid-19-patientsin-leishenshan-hospital-wuhan-china 